\title{
DESCRIÇÃO DE DUAS NOVAS ESPÉCIES DE SCIAENIDAE (PERCIFORMES) DE ÁGUA DOCE DA BACIA AMAZÔNICA
}

\author{
Leoneza SOARES ${ }^{1}$, Lilian CASATTI ${ }^{2}$
}

\begin{abstract}
RESUMO - São descritas duas novas espécies de Sciaenidae da região Amazônica, pertencentes aos gêneros Pachyurus e Plagioscion. Pachyurus junki sp. n. difere das demais espécies congenéricas descritas por apresentar a seguinte combinação de caracteres: extremidade posterior da nadadeira pélvica distante do ânus; largura interorbital contida menos do que 6,0 vezes no comprimento da cabeça; segundo espinho da nadadeira anal contido mais do que 2,1 vezes no comprimento da cabeça; 29 a 33 raios na nadadeira dorsal; 11 a 16 séries de escamas acima da linha lateral; 11 a 16 séries de escamas abaixo da linha lateral e 58 a 68 escamas perfuradas na linha lateral. Plagioscion montei sp. $\mathrm{n}$. difere das demais espécies do gênero por apresentar: ânus distante da nadadeira anal, estando a distância ânus-anal contida de 2,4 a 3,5 vezes no comprimento da cabeça; nadadeira peitoral relativamente longa, quando adpressa sua extremidade posterior chega próximo ou ultrapassa a linha vertical que passa pela origem do ânus; largura interorbital relativamente ampla, contida entre 3,5 e 5,0 vezes no comprimento da cabeça e segundo espinho da nadadeira anal forte e curto, contido de 2,5 a 4,4 vezes no comprimento da cabeça.
\end{abstract}

Palavras-chave: Pachyurus, Plagioscion, Sciaenidae, nova espécie, bacia Amazônica.

Description of Two New Species of Freshwater Sciaenidae (Perciformes) from Amazon River Basin.

ABSTRACT - Two new species of freshwater Sciaenidae genera Pachyurus and Plagioscion are described from Amazon River basin. Pachyurus junki sp. $\mathrm{n}$. differs from its congeners by the following characters combination: posterior tip of pelvic fin distant from the anus, interorbital width less than 6.0 times in the head length, second anal fin spine more than 2.1 times in the head length, 29 to 33 soft rays in the dorsal fin, 11 to 16 rows of scales above to lateral line, 11 to 16 rows of scales below, and 58 to 68 perfored scales in the lateral line. Plagioscion montei sp. $\mathrm{n}$. differs from its congeners by having: anus distant from the anal fin, the distance anus-anal fin from 2.4 to 5.0 times in the head length; pectoral fin relatively long, when adpressed its posterior tip reaches or surpasses the vertical line that crosses the anus; interorbital width relatively large, from 3.5 to 4.9 times in the head length, and second anal fin spine strong and short, from 2.5 to 4.4 times in the head length.

Key-words: Pachyurus, Plagioscion, Sciaenidae, new species, Amazon River Basin.

\section{INTRODUÇÃO}

A familia Sciaenidae (pescadas, corvinas, betaras) inclui aproximadamente 78 gêneros e 287 espécies que ocorrem em águas costeiras, estuarinas e doces de áreas tropicais e temperadas (Chao, 1986).
Cinco de seus gêneros são restritos à água doce, podendo ocorrer também em estuários: Aplodinotus Rafinesque, Pachypops Gill, Pachyurus Agassiz, Plagioscion Gill e Boesemania Trewavas. Destes, Aplodinotus ocorre nas Américas do Norte e Central (Chao, 1978); Pachypops, Pachyurus

\footnotetext{
'Laboratório de Ictiologia, Departamento de Oceanografia e Limnologia, UFRN, Praia da Mãe Luiza, s/n, Natal, RN, Brasil.

${ }^{2}$ Laboratório de Ictiologia, Departamento de Biologia, FFCLRP-USP, Av. Bandeirantes, 3900, 14040-901, Ribeirão Preto, SP, Brasil. E-mail: licasatti@netsitemail.com.br
} 
e Plagioscion na América do Sul (Chao, 1978) e Boesemania na Tailândia, Vietnã, Camboja e Sumatra (Kottelat et al., 1993). Outras espécies que ocorrem no Indo-Pacífico, pertencentes aos gêneros Dendrophysa Trewavas, Johnius Bloch e Nibea Jordan \& Thompson, eventualmente podem ser coletadas em água doce (Kunio Sazaki, comunicação pessoal).

Soares. (1978), em sua Dissertação de Mestrado, revisando as espécies de Sciaenidae da região Amazônica brasileira, propôs novos nomes para duas novas espécies de Pachyurus e Plagioscion. Entretanto, a aplicação dos Artigos 8 e 9 do Código Internacional de Nomenclatura Zoológica (ICZN, 1999), invalida a descrição de novos táxons em Dissertações e obras similares. Sendo assim, nosso objetivo é descrever apropriadamente os novos táxons de Soares (1978), disponibilizando os nomes propostos pela autora.

\section{MATERIAL E MÉTODOS}

Os espécimes examinados (incluindo material adicional comparativo e tipos) estão depositados em 16 instituições (os acrônimos seguem Leviton et al., 1985). Foram verificados 22 caracteres morfométricos e nove merísticos, de acordo com a metodologia descrita por Hubbs \& Lagler (1958). A medida da largura interorbital foi efetuada na altura da borda anterior da pupila. As contagens do número de escamas da linha lateral incluem aquelas perfuradas até o limite posterior da placa hipural. $\mathrm{Na}$ contagem dos rastros branquiais foram incluidos todos os rastros do primeiro arco branquial do lado esquerdo do corpo. As medidas corporais foram interpretadas como proporções do comprimento padrão, exceto o comprimento do segundo espinho da nadadeira anal e a distância ânus-anal; estas, juntamente com as medidas cefálicas, foram interpretadas como proporções do comprimento da cabeça. Nas diagnoses e descrições os valores entre colchetes se referem às modas no caso de variável merística e, às médias, no caso de variável morfométrica.

Pachyurus junki sp. n. (Fig. 1, Tab. 1)

Material examinado: HolótiPo INPA 619 (185,0 mm), Rio Solimões, Coari, AM, Brasil, col. Equipe de Ictiologia do INPA, 22 abril/1979;

PARÁtIPOS: MZUSP 37572 (226,0 $\mathrm{mm})$, Rio Uraricoera, Estação Ecológica de Maracá, RR; CAS 32058 (207,0 mm), Rio Tapajós, Santarém, PA, col. C. Ternetz, setembro/1924; MCP 15121 (02 ex., 199,0-201,0), Rio Tapajós, Itaituba, col. C. Lucena, novembro/1991; INPA 4049 (05 ex., 176,0-293,0 mm), Rio Xingu, Arroz Cru, PA, col. L.R. Py-Daniel \& J. Zuanon, 01 outubro/1990.

Diagnose: Difere de Pachyurus paucirastrus e $P$. bonariensis por apresentar a nadadeira pélvica relativamente curta, não atingindo o ânus; de $P$. squamipinnis por apresentar largura interorbital contida menos do que $6,0[4,43]$ vezes no comprimento da cabeça $(5,4$ a $7,0 \mathrm{em}$ $P$. squamipinnis); de $P$. francisci por apresentar o segundo espinho da nadadeira anal contido mais do que 2,1 


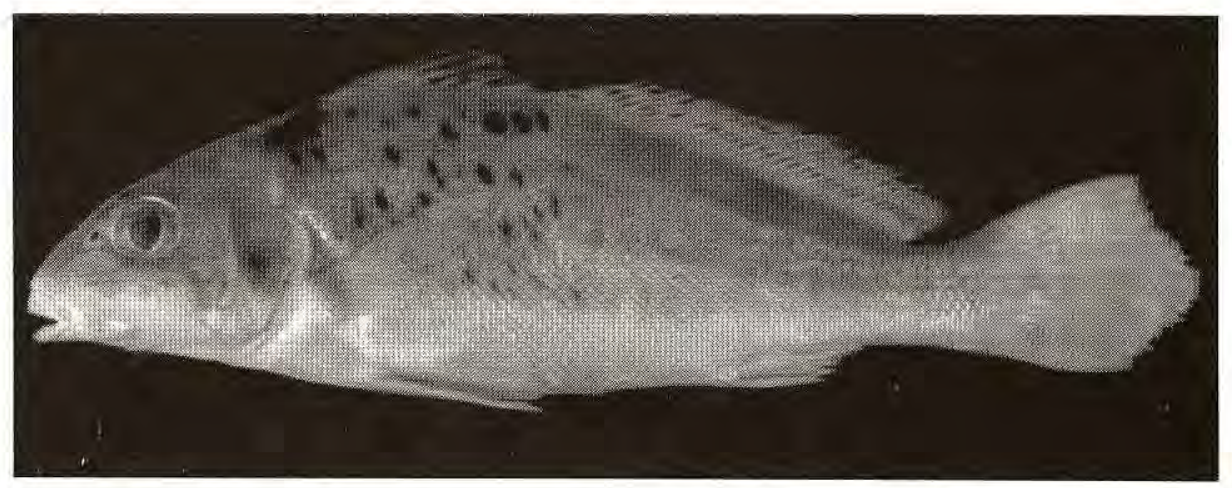

Figura 1. Pachyurus junki sp. n., holótipo, INPA 619, 185,0 mm CP.

$[2,45]$ vezes no comprimento da cabeça (1,5 a 1,8 em $P$. francisci); de P. schomburgkii por apresentar de 29 a 33 [33] raios na nadadeira dorsal (23 a 28 [27] em P. schomburgkii), 11 a 16 [12] séries de escamas acima da linha lateral $(9$ a 11 [10] em $P$. schomburgkii), 11 a 16 [13] séries de escamas abaixo da linha lateral ( 9 a 12 [10] em P. schomburgkii) e de 58 a 68 [62] escamas perfuradas na linha lateral (49 a 54 [53] em P. schomburgkii).

$\mathrm{D}$ e s c ri ç ã o : D a d o s morfométricos e merísticos dos exemplares examinados na Tabela 1. Corpo relativamente alongado, algo comprimido lateralmente, maior altura do corpo na altura da primeira nadadeira dorsal. Perfil dorsal convexo. Perfil ventral reto até a região pré-nadadeira anal. Cabeça pontiaguda, perfil dorsal formando um ângulo de aproximadamente $45^{\circ} \mathrm{com}$ o perfil ventral da cabeça. Boca pequena e inferior, 5 a 6 poros mentonianos evidentes a olho nu. Abertura bucal não alcançando a linha vertical que passa pela margem ante- rior da órbita. Dentes de ambas as maxilas pequenos e dispostos em faixas viliformes. Focinho pontiagudo, seu comprimento aproximadamente igual ao diâmetro horizontal do olho. Narinas de cada lado bem próximas, circulares anteriormente, alongadas posteriormente. Olho elíptico de tamanho moderado, seu diâmetro horizontal contido de 2,5 a 3,8 [3,22] vezes no comprimento da cabeça. Canais da linha lateral passando pelos lacrimais, infraorbitais, pré-opérculo e maxila inferior claramente visíveis externamente. Margem do préopérculo suavemente denticulada. Primeiro arco branquial esquerdo com $17-20$ [19] rastros branquiais curtos e largos. Escamas ctenóides, exceto no focinho, pré-opérculo e membranas inter-radiais das nadadeiras dorsal, anal e caudal, onde são ciclóides. Linha lateral estendendo-se até a extremidade da nadadeira caudal; 58 a 68 [62] escamas perfuradas até o final da placa hipural. De 11 a 16 [12] séries de escamas acima e 11 a 16 [13] séries de escamas abaixo da linha lateral. 
Primeira nadadeira dorsal com dez espinhos, o primeiro espinho pequeno; segunda dorsal com um espinho seguido por 29 a 33 [33] raios moles, separada da primeira dorsal por um sulco. Nadadeira peitoral com 16 a 18 [18] raios moles, inserida um pouco à frente da vertical que cruza $o$ primeiro espinho da dorsal. Nadadeira pélvica inserida um pouco atrás da vertical que cruza a base da peitoral, seu primeiro raio mole não atinge o ânus. Primeiro espinho da nadadeira anal reduzido, o segundo relativamente bem desenvolvido, seu comprimento contido de 2,1 a 3,5 $[2,45]$ vezes no comprimento da cabeça, seguido por 6 a 7 [7] raios moles. Nadadeira caudal larga e de forma romboidal, com 17 raios principais $(9+8)$.

Bexiga natatória com uma vesícula central simples e um par de pequenos apêndices em sua porção anterior, não ramificados (Fig. 2 a).

Coloração em álcool: Porção dorsal da cabeça e região pré-dorsal castanhas; $3 / 4$ superiores do corpo castanho-amarelados; $1 / 4$ inferior amarelo-prateado; pequenas manchas
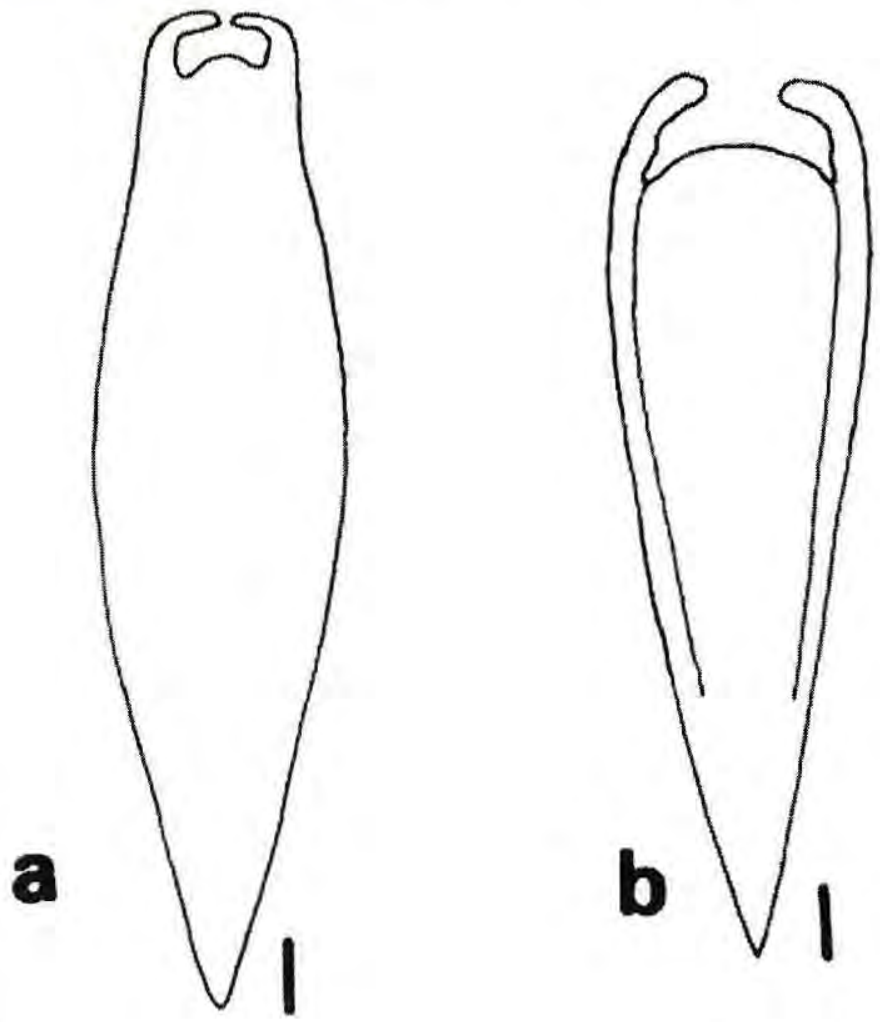

Figura 2. Vista ventral das bexigas natatórias de: a) Pachyurus junki sp. n.; b) Plagioscion montei sp. n. (barra $=5 \mathrm{~mm}$ ). 
castanho-escuras arredondadas, de tamanho um pouco maior do que o das aberturas nasais, distribuidas irregularmente na porção látero-dorsal do corpo (ausentes em jovens de até aproximadamente $80 \mathrm{~mm}$ de comprimento padrão). Borda da primeira nadadeira dorsal escurecida; membrana inter-radial da primeira e segunda dorsais com pequenos cromatóforos escuros, na segunda dorsal duas a três listras longitudinais acinzentadas. Nadadeiras peitoral, pélvica e anal amarelo-claras.

Distribuição: Rios da bacia Amazônica (Fig. 3).

Etimologia: O nome específico "junki" é uma homenagem ao Dr. Wolfgang Johannes Junk, Coordenador do Curso de Biologia de Água Doce e Pesca Interior do Instituto Nacional de Pesquisas da Amazônia no ano de 1978.

Comentários: Essa espécie é

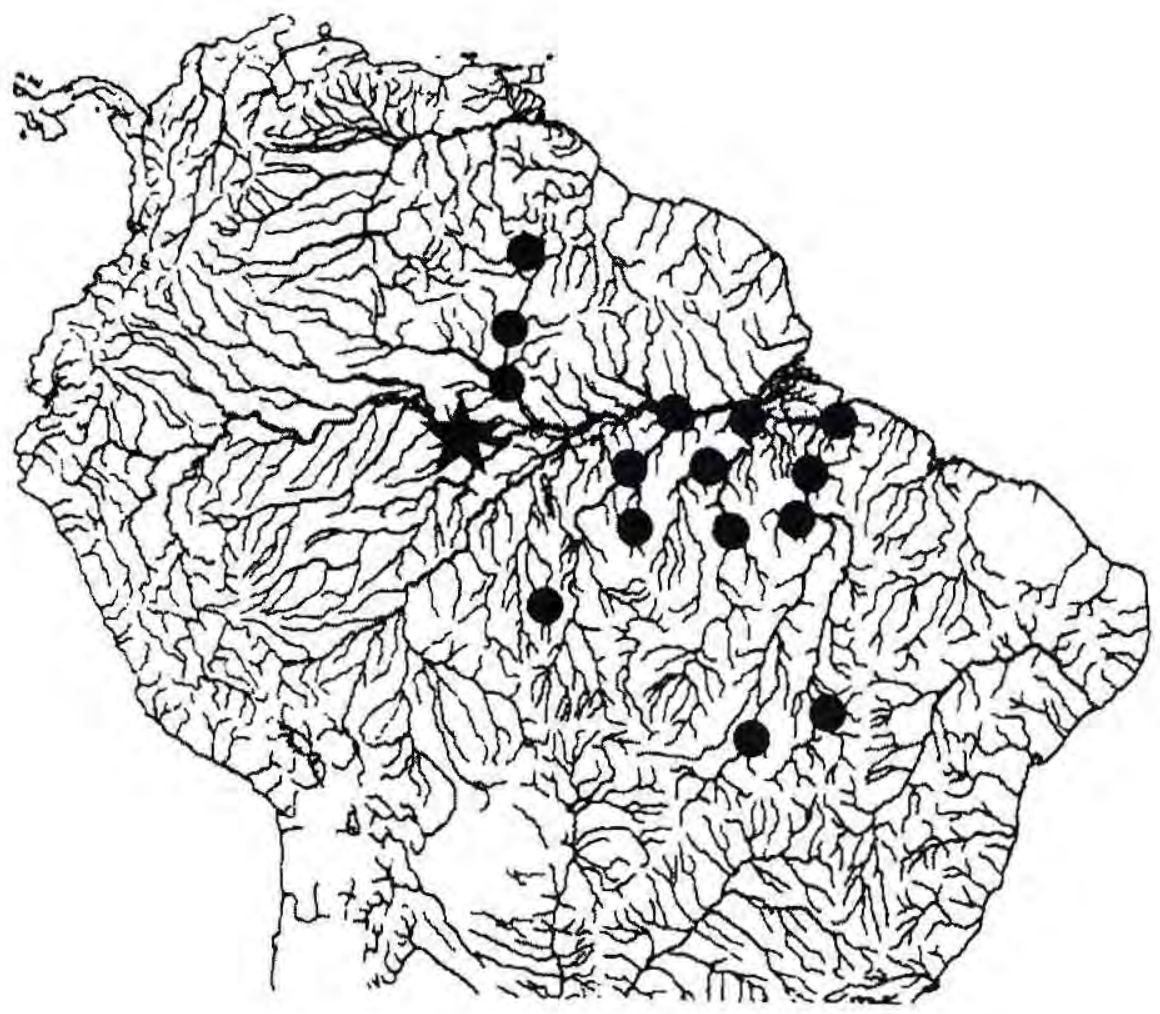

Figura 3. Distribuição geográfica de Pachyurus junki sp. n.; estrela indica a localidade onde o holótipo foi coletado, demais símbolos representam mais de um lote examinado ou localidade de coleta. 
morfometricamente semelhante a Pachyurus schomburgkii, com a qual ocorre em simpatria e difere meristicamente por possuir maior número de séries transversais de escamas e maior número de raios moles na nadadeira dorsal (Fig. 4). Além disso, a nova espécie possui pequenas manchas castanho-escuras distribuídas por toda a lateral do corpo, enquanto que em $P$. schomburgkii as manchas estão restritas à região imediatamente abaixo da primeira nadadeira dorsal.

Plagioscion montei sp.n. (Fig. 5, Tab. 2)

Material examinado: HolótIPO INPA 15959 (213,0 $\mathrm{mm})$, Rio Solimões, Lago Janauacá, AM, Brasil; PARÁtiPOS: INPA 604 (18 ex., 155,9-284,0 mm), coletados com o holótipo; MZUSP 34086 (207,0 mm), Rio Madeira, Calama, RO, col. M. Goulding, junho/1980; MZUSP 34090 (03 ex., 175,9-182,6 mm), Rio Tapajós, entre Itaituba e São Luis, PA,

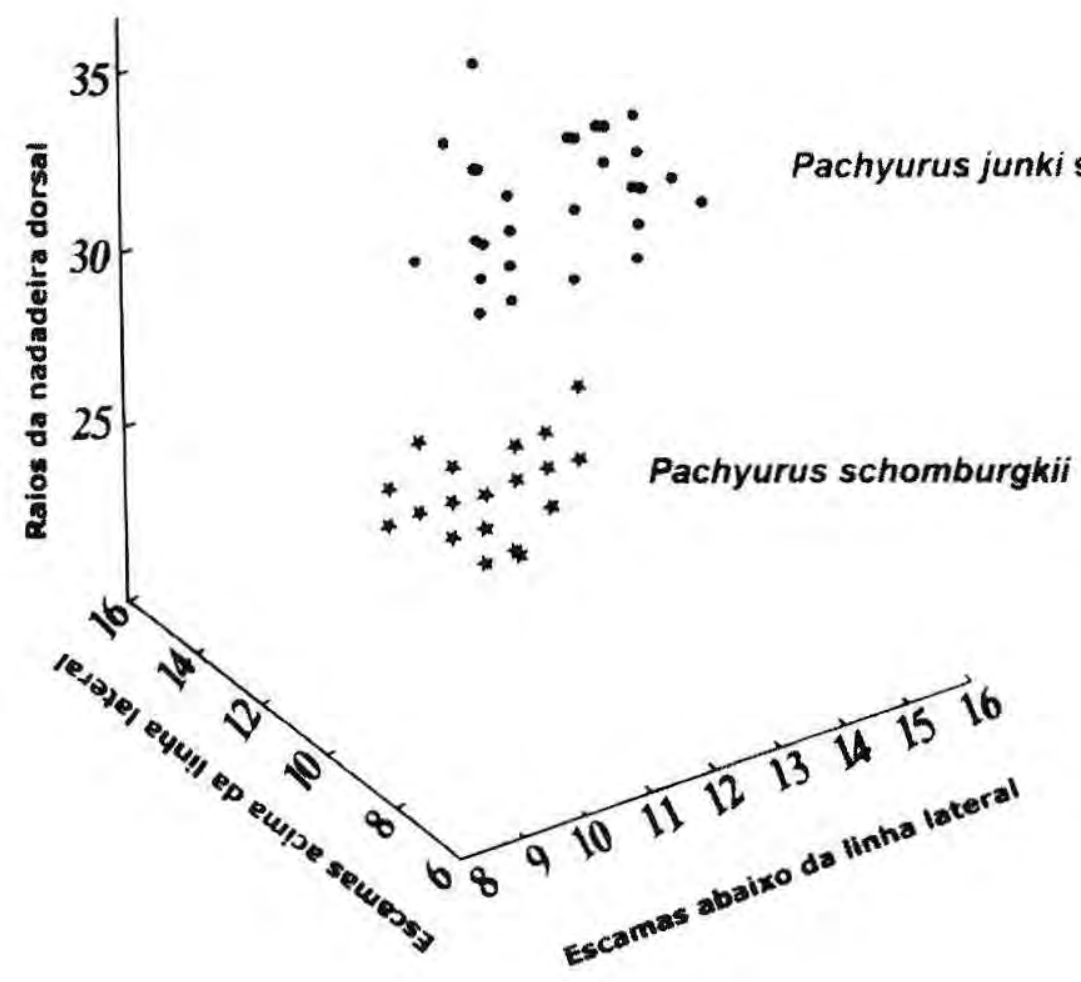

Figura 4. Número de raios moles da segunda nadadeira dorsal contra o número de fileiras de escamas acima e abaixo da linha lateral de Pachyurus schomburgkii (estrelas) e Pachyurus junki sp. n. (circulos). 
Tabela 1. Biometria do holótipo, 09 parátipos e outros 24 exemplares de Pachyurus junki sp. $\mathrm{n}$. Os cálculos estatísticos incluem todo o material examinado.

\begin{tabular}{lcccc}
\hline & Holótipo & Parátipos & $\begin{array}{c}\text { Outros } \\
\text { exemplares }\end{array}$ & $\begin{array}{c}\text { Média, desvio } \\
\text { padrăo e moda* }\end{array}$ \\
\hline Comprimento padrăo $(\mathrm{mm})$ & 185,0 & $176,0-293,0$ & $66,2-280,7$ & $183,45 \pm 55,38$
\end{tabular}

Proporçōes do comprimento padrăo:

Maior altura do corpo

Distância pré-dorsal

Base da nadadeira dorsal

Distância do rsal-caudal

Distância pré-pélvica

Distância pré-ânus

Distância pré-anal

Base da nadade ira anal

Distância anal-caudal

Comprimento da nadadeira dorsal

Comprimento da nadadeira peitoral

Comprimento da nadadeira pélvica

Distância pélica-ânus

Atura do pedínculo caudal

Comprimento da cabeça

$\begin{array}{cccc}3,4 & 3,5-3,8 & 3,3-4,6 & 3,80 \pm 0,29 \\ 2,7 & 2,7-2,8 & 2,4-2,7 & 2,81 \pm 0,15 \\ 1,6 & 1,7-1,8 & 1,6-1,8 & 1,71 \pm 0,09 \\ 9,3 & 8,2-9,1 & 8,5-10,7 & 8,92 \pm 0,64 \\ 2,9 & 2,9 & 2,9-3,7 & 2,94 \pm 0,22 \\ 1,7 & 1,6-1,7 & 1,6-2,1 & 1,72 \pm 0,10 \\ 1,4 & 1,4 & 1,4-1,6 & 1,52 \pm 0,33 \\ 10,8 & 11,5-13,6 & 10,9-14,5 & 11,92 \pm 1,20 \\ 4,5 & 4,0-4,5 & 3,7-4,7 & 4,34 \pm 0,63 \\ 5,9 & 5,7-6,2 & 5,4-6,7 & 5,88 \pm 0,49 \\ 5,0 & 4,6-5,1 & 4,4-5,6 & 4,91 \pm 0,34 \\ 4,8 & 5,3-5,5 & 4,6-5,9 & 5,07 \pm 0,37 \\ 3,9 & 3,9-4,5 & 3,8-4,5 & 4,08 \pm 0,21 \\ 10,3 & 10,4-12,0 & 10,1-13,1 & 11,40 \pm 0,81 \\ 3,4 & 3,4-3,6 & 3,1-4,5 & 3,48 \pm 0,26\end{array}$

Proporçōes do comprimerto da cabeça:

Comprimento do focinho

Diâmetro horizontal do olho

$\begin{array}{llll}2,7 & 2,6-2,9 & 2,5-3,4 & 2,80 \pm 0,25 \\ 3,5 & 3,3-3,5 & 2,5-3,8 & 3,22 \pm 0,33 \\ 2,5 & 2,4-2,6 & 2,4-4,0 & 2,66 \pm 0,38 \\ 3,9 & 4,0-4,5 & 4,0-6,0 & 4,43 \pm 0,52 \\ 5,8 & 5,8-8,6 & 4,5-8,6 & 6,69 \pm 0,76 \\ 2,5 & 2,2-2,8 & 2,1-3,5 & 2,45 \pm 0,33\end{array}$

Comprimento do segundo espinho da anal

Contagens:

Escamas da linha lateral

Fileira de escamas acima da linha lateral

Fileira de escamas abaixo da linha lateral

Elementos da nadadeira dorsal

Elementos da nadadeira anal

Elementos da nadadeira pélvica

Elementos da nadadeira peitoral

Poros ra mandibula

Rastros branquiais

\begin{tabular}{cccc}
61 & 5961 & 5868 & 62 \\
12 & $12-14$ & $11-16$ & 12 \\
12 & $12-15$ & $11-16$ & 13 \\
$X 1+32$ & $X I+29-33$ & $X I+29-33$ & $X 1+33$ \\
$\|+7$ & $\| 1+6-7$ & $\|+6-7$ & $\|+7$ \\
$1+5$ & $1+5$ & $1+5$ & $1+5$ \\
$1+16$ & $1+16-18$ & $1+16-18$ & $1+18$ \\
5 & 5 & $5-6$ & 6 \\
14 & $16-20$ & $17-20$ & 19 \\
\hline
\end{tabular}

- calculada para as contagens 


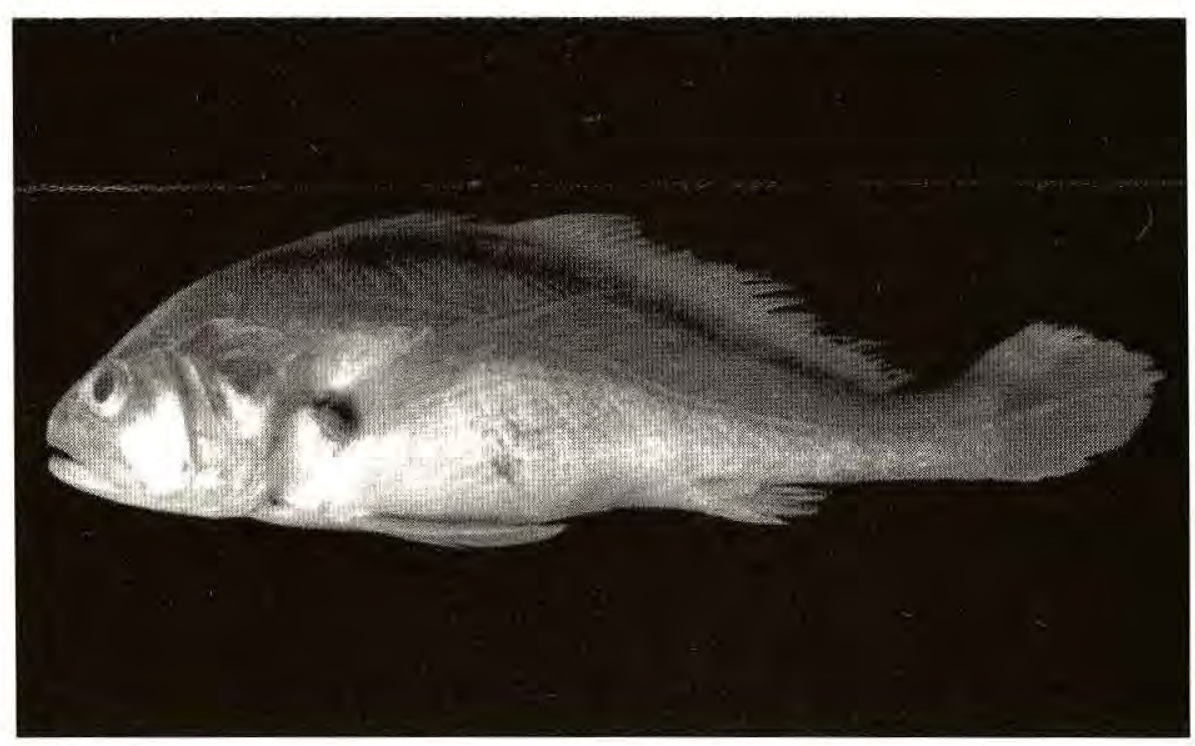

Figura 5. Plagioscion montei sp. n., parátipo, MZUSP 34086, 207,0 mm CP.

col. M. Goulding, setembro/1983.

Diagnose: Difere de $P l$. squamosissimus e Pl. ternetzi por apresentar o ânus localizado distante da nadadeira anal, estando a distância ânus-anal contida de 2,4 a 3,5 [2,94] vezes no comprimento da cabeça (de 3,4 a 5,6 vezes no comprimento da cabeça em $P l$. squamosissimus e $P l$. ternetzi); de $P l$. auratus por apresentar a nadadeira peitoral relativamente longa, quando adpressa ao corpo sua extremidade posterior chega próximo ou ultrapassa a linha vertical que passa pela origem do ânus; e também por apresentar olho mais desenvolvido, seu diâmetro horizontal contido de 3,8 a $5,2[4,43]$ vezes no comprimento da cabeça (de 5,0 a 6,2 em Pl. auratus); de $P l$. surinamensis por apresentar largura interorbital mais ampla, contida entre 3,5 e $4,9[4,42]$ vezes no comprimento da cabeça, e segundo espinho da nadadeira anal forte e curto, contido de 2,5 a 4,4 [3,35] vezes no comprimento da cabeça (em $\mathrm{Pl}$. surinamensis a largura interorbital é mais estreita, contida de 5,0 a 6,5 vezes no comprimento da cabeça e o espinho da anal é forte e longo, contido de 1,5 a 2,8 vezes no comprimento da cabeça).

Descrição: Dados morfométricos e merísticos na Tabela 2. Corpo relativamente alongado, algo comprimido, maior altura do corpo na origem da nadadeira dorsal. Perfil dorsal convexo. Perfil ventral reto da região pré-pélvica até a origem da nadadeira anal. Perfil anterior da cabeça suavemente arredondado. Boca terminal, oblíqua em vista lateral. Abertura bucal não alcançando a linha vertical que passa pela margem posterior da órbita. Lábios carnosos. Maxilas protráteis, com papilas 
viliformes cercadas por fileiras irregulares de dentes cônicos de tamanhos variados. Comprimento do focinho semelhante ao do diâmetro horizontal do olho. Olho arredondado de tamanho moderado, seu diâmetro horizontal contido de 3,8 a $5,2[4,43]$ vezes no comprimento da cabeça. Narinas de cada lado da cabeça próximas entre si; abertura anterior circular, posterior em forma de crescente. Canais da linha lateral visiveis externamente na cabeça, passando pelos lacrimais, infraorbitais, pré-opérculo e maxila inferior. Margem do pré-opérculo suavemente denticulada, opérculo com a borda superior pontiaguda, membrana opercular bem desenvolvida, atingindo a base da peitoral. Primeiro arco branquial esquerdo com 19 a 23 [21] rastros branquiais relativamente bem desenvolvidos. Escamas ctenóides, exceto na região cefálica, pré-opérculo e membranas inter-radiais das nadadeiras dorsal, anal e caudal, onde são ciclóides. Linha lateral curvada até o terço anterior da segunda nadadeira dorsal; escamas da linha lateral grandes, cobertas parcialmente por escamas pequenas, estendendo-se até a extremidade dos raios medianos da nadadeira caudal; 48 a 54 [50] escamas perfuradas na linha lateral; 8 a 10 [10] séries transversais de escamas acima e 9 a 11 [9] séries de escamas abaixo da linha lateral.

Primeira nadadeira dorsal com dez espinhos, o primeiro pequeno; segunda dorsal com um espinho e 31 a 39 [34] raios moles, separada da primeira dorsal por um sulco.
Nadadeira peitoral com 16 a 17 [16] raios moles, inserida levemente à frente do primeiro espinho da primeira dorsal; sua extremidade posterior alcança ou ultrapassa a linha vertical que passa pelo ânus. Nadadeira pélvica inserida um pouco atrás da vertical que cruza a base da peitoral; seu primeiro raio mole não atinge o ânus. Primeiro espinho da nadadeira anal reduzido, o segundo relativamente forte e curto (seu comprimento cerca de $3 / 4$ do comprimento do maior raio mole da anal e contido de 2,4 a 4,4 vezes no comprimento da cabeça), seguido por 6 a 7 [6] raios moles. Ânus distante da nadadeira anal, a distância ânus-anal contida de 2,4 a $3,5[2,94]$ vezes no comprimento da cabeça. Nadadeira caudal larga e de forma romboidal, com 17 raios principais $(9+8)$; nos jovens os raios medianos (último do lobo superior e primeiro do lobo inferior) são mais longos do que os demais.

Bexiga natatória formada por uma vesícula central bem desenvolvida, de onde sai, anteriormente, um par de apêndices em forma de cornos

(Fig. 2 b).

Coloração em álcool: Cabeça e porção látero-dorsal do corpo castanho escuro; $01 / 4$ inferior do corpo amarelo claro. Borda e membrana inter-radial da primeira dorsal, segunda dorsal e peitoral com inúmeros cromatóforos marrons, o que confere a estas nadadeiras um colorido escuro. Alguns exemplares (aproximadamente $40 \%$ do material examinado), ocasionalmente em um mesmo lote, 
podem apresentar as nadadeiras peitorais claras. Peitoral com mancha axilar de cor marrom escura. Pélvica e anal com cromatóforos escuros irregularmente distribuídos.

Distribuição: Rios da bacia Amazônica, Brasil e Peru (Fig. 6).

Etimologia: O nome específico "montei" é uma homenagem ao Prof. Sebastião Monte, Chefe do Departamento de Oceanografia e Limnologia da Universidade Federal do Rio Grande do Norte no ano de 1978.
Comentários: O epíteto Plagioscion montei (grafado como $\mathrm{Pl}$. "monti") foi utilizado por Worthmann $(1980,1983,1987,1992)$ e Worthmann \& Oliveira (1987) em trabalhos que comparam fatores de crescimento entre essa espécie e $P l$. squamosissimus. Entretanto, nenhuma dessas publicações fornecem os caracteres que diferenciam $\mathrm{Pl}$. montei das demais do gênero; ou seja, mesmo a citação de $P l$. "monti" nos trabalhos de Worthmann não disponibiliza o nome proposto por Soares (1978),

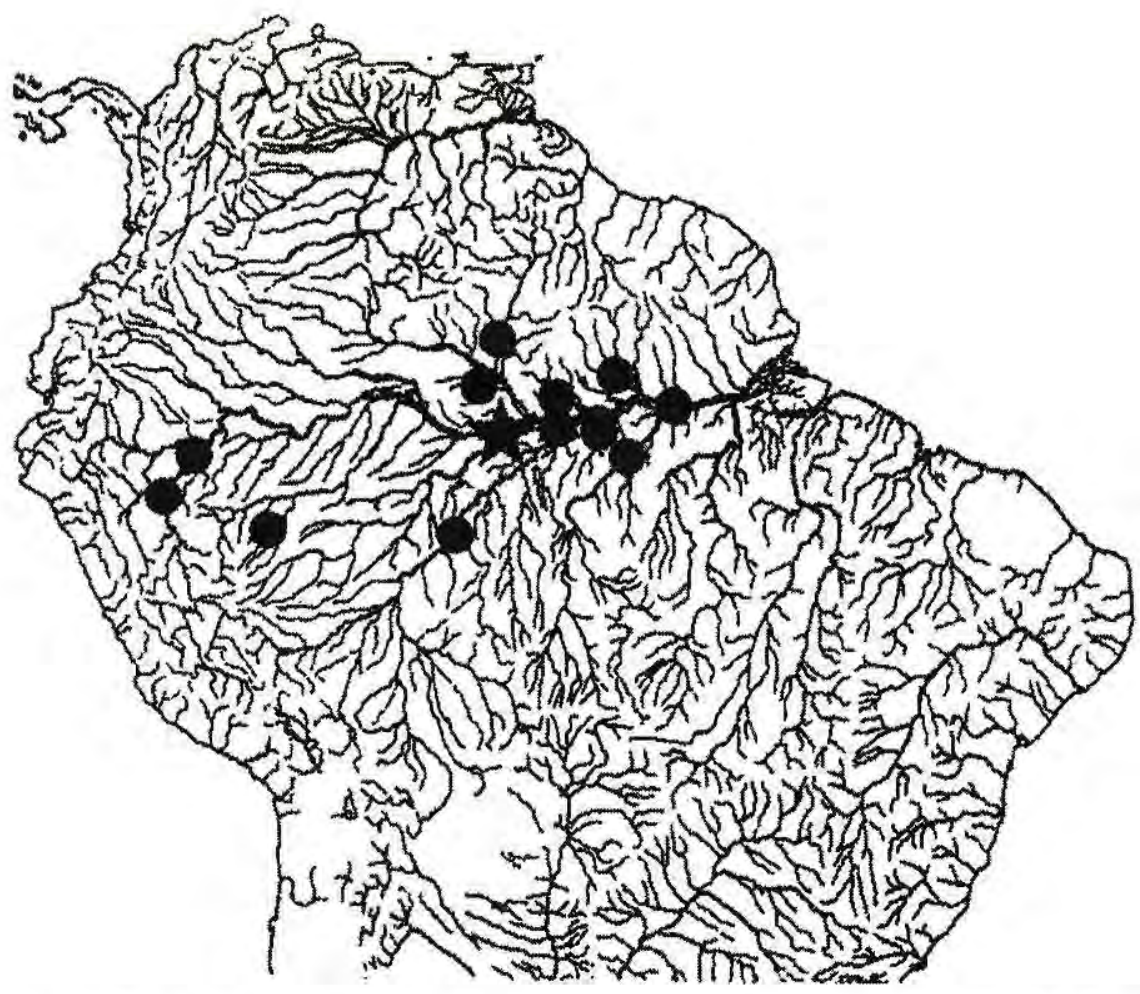

Figura 6. Distribuição geográfica de Plagioscion montei sp. n.; estrela indica a localidade onde o holótipo foi coletado, demais símbolos representam mais de um lote examinado ou localidade de coleta. 
Tabela 2. Biometria do holótipo, 21 parátipos e outros 73 exemplares de Plagioscion montei sp. $\mathrm{n}$. Os cálculos estatísticos incluem todo o material examinado.

\begin{tabular}{|c|c|c|c|c|}
\hline & Holótipo & Parátipos & $\begin{array}{l}\text { Outros } \\
\text { exemplares }\end{array}$ & $\begin{array}{l}\text { Média, desvio } \\
\text { padrão e moda* }\end{array}$ \\
\hline Comprimerto padrāo (mm) & 213,0 & $155,9-284,0$ & $78,0-262,2$ & $187,70 \pm 39,81$ \\
\hline \multicolumn{5}{|l|}{ Proporçōes do comprimento padrăo: } \\
\hline Maior altura do corpo & 3,4 & $3,3-3,6$ & $3,2-4,1$ & $3,48 \pm 0,19$ \\
\hline Distância pré-dorsal & 2,9 & $2,7-3,0$ & $2,7-3,1$ & $2,88 \pm 0,09$ \\
\hline Base da nadadeira dorsal & 1.7 & $1,6-1,8$ & $1,6-1,9$ & $1,64 \pm 0,05$ \\
\hline Distância dorsal-caudal & 10,5 & $8,6-10,1$ & $8,5-10,8$ & $9,35 \pm 0,58$ \\
\hline Distância pré-pélvica & 2,9 & $2,8-3,1$ & $2,7-3,1$ & $2,89 \pm 0,08$ \\
\hline Distância pré-ânus & 1,6 & $1,6-1,8$ & $1,5-1,7$ & $1,66 \pm 0,07$ \\
\hline Distância pré-anal & 1,4 & $1,4-1,5$ & $1,3-1,5$ & $1,44 \pm 0,26$ \\
\hline Distância pélica-ânus & 3,5 & $3,4-3,8$ & $3,2-4,4$ & $3,75 \pm 0,27$ \\
\hline Distância pélvica-anal & 2,6 & $2,5-2,8$ & $2,4-2,9$ & $2,63 \pm 0,11$ \\
\hline Base da radade ira anal & 13,1 & $12,6-14,9$ & $11,9-17,0$ & $13,79 \pm 1,03$ \\
\hline Distância anal-caudal & 4,2 & $3,7-4,2$ & $3,8-4,7$ & $4,19 \pm 0,19$ \\
\hline Comprimento da nadadeira dorsal & 7,5 & $7,3-8,0$ & $6,7-10,8$ & $8,19 \pm 0,94$ \\
\hline Comprimerto da nadadeira peitoral & 3,5 & $2,7-3,6$ & $2,7-4,1$ & $3,61 \pm 0,24$ \\
\hline Comprimento da nadadeira pélvica & 4,4 & $3,9-5,2$ & $3,8-5,9$ & $4,51 \pm 0,40$ \\
\hline Altura do pedúnculo caudal & 11,3 & $10,9-12,7$ & $10,3-13,9$ & $11,87 \pm 0,70$ \\
\hline Comprimento da cabeça & 3,2 & $2,8-3,2$ & $2,9-3,6$ & $3,16 \pm 0,12$ \\
\hline \multicolumn{5}{|l|}{ Proporọoes do comprimerto da cabeça: } \\
\hline Distância ânus-anal & 3,3 & $2,8-3,3$ & $2,4-3,5$ & $2,94 \pm 0,32$ \\
\hline Comprimento do focinho & 4,0 & $4,1-5,0$ & $3,6-5,0$ & $4,23 \pm 0,24$ \\
\hline Diâmetro horizontal do olho & 4,9 & $4,1-5,0$ & $3,8-5,2$ & $4,43 \pm 0,37$ \\
\hline Distância pós-orbital & 1,7 & $1,6-1,8$ & $1,6-1,8$ & $1,73 \pm 0,06$ \\
\hline Largura interorbital & 4,1 & $4,4-5,0$ & $3,5-4,9$ & $4,42 \pm 0,28$ \\
\hline Largura da fenda bucal & 3,5 & $3,4-3,8$ & $2,9-3,7$ & $3,42 \pm 0,26$ \\
\hline Comprimento do segundo espinho da anal & 3,2 & $2,8-3,9$ & $2,5-4,4$ & $3,35 \pm 0,47$ \\
\hline \multicolumn{5}{|l|}{ Contagens: } \\
\hline Escamas da linha lateral & 50 & 48.50 & 48.54 & 50 \\
\hline Fileira de escamas acima da linha lateral & 8 & $8-10$ & $8-10$ & 10 \\
\hline Fileira de escamas abaixo da linha lateral & 9 & $9-10$ & $9-11$ & 9 \\
\hline Elementos da nadadeira dorsal & $X 1+35$ & $X I+3237$ & $\mathrm{XI}+31.39$ & $X I+34$ \\
\hline Elementos da nadadeira anal & $\|+6$ & $\|+6-7$ & $11+6-7$ & $\|+6$ \\
\hline Elementos da nadadeira pélvica & $1+5$ & $1+5$ & $1+5$ & $1+5$ \\
\hline Elementos da nadadeira peitoral & $1+16$ & $1+16-17$ & $1+16-17$ & $1+16$ \\
\hline Rastros branquiais & 20 & $19-22$ & 1923 & 21 \\
\hline
\end{tabular}

* calculada para as contagens 
conforme o Capítulo 4 do ICZN (1999).

\section{AGRADECIMENTOS}

Expressamos nossos agradecimentos ao Prof. Sebastião Monte, chefe do Departamento de Oceanografia e Limnologia (UFRN) pelo apoio; aos Drs. E. Ferreira, L.R. Py-Daniel, J. Zuanon, J.L. de Figueiredo, H.A. Britski, O.T. Oyakawa, R.E. Reis, C.A. Lucena, R. Barthem, H. Higuchi, W. Eschmeyer, D. Catania, B. Chernoff, M.A. Rogers, K. Swagel, K. Hartel, W. Saul; R.P. Vari, S. Jewett, B. Brown, M. Carvalho, A. Gill, P. Campbell, M. Van Oijen, E. Mikschi, H. Wellendorf, S. Müller, J. Montoya, P. Pruvost e M. Jégu, pelo apoio durante visitas às suas instituições; ao Dr. L.N. Chao pela permissão para consulta de literatura e R.C. Benine pela leitura crítica do manuscrito. A primeira autora é docente no Departamento de Oceanografia e Limnologia da UFRN; a segunda autora é Pós-Doutoranda no Departamento de Biologia da FFCLRP-USP e recebe apoio financeiro da FAPESP.

\section{Bibliografia citada}

Chao, L.N. 1978. A basis for classifying western Atlantic Sciaenidae (Teleostei: Perciformes). NOAA Technical Report NMFS Circular 415, Washington, $65 \mathrm{pp}$.

Chao, L.N. 1986. A synopsis on zoogeography of the Sciaenidae. Pp. 570-589 In: T. Uyeno, R. Arai, T. Taniuchi, K.Matsuura (Eds.). Indo-Pacific fish biology: Proceedings of the Second International Conferecence of Indo-Pacific Fishes. Ichthyological Society of Japan, Tokyo.

Hubbs, C.L.; Lagler, K.F. 1958. Fishes of the
Great Lakes region. Univ. Michigan Press, Michigan, $213 \mathrm{pp}$.

ICZN. 1999. International Code of Zoological Nomenclature. International Trust for Zoological Nomenclature, London, 306 pp.

Kottelat, M.; Whitten, A.J.; Kartikasari, S.N.; Wirjoatmodjo, S. 1993. Freshwater fishes of Indonesia and Sulawesi. Periphus Ed., Hong Kong, 221 pp.

Leviton, A.E.; Gibbs Jr., R.H.; Heal, E.; Dawson, C.E. 1985. Standards in herpetology and ichthyology: Part I. Standard symbolic codes for institutional resource collections in herpetology and ichthyology. Copeia, 1985(3): 802-832.

Soares, L.H. 1978. Revisão taxonômica dos sciaenideos de água doce da região Amazônica brasileira. Dissertação de Mestrado, Instituto Nacional de Pesquisas da Amazônia, 72 pp.

Worthmann, H.O. 1980. The early growth stages of the pescada (Plagioscion monti Soares). Arch. Fish. Wiss., 30(2/3): 145-156.

Worthmann, H.O. 1983. A comparative study of the growth of the postlarval and juvenile Pescadas Plagioscion squamosissimus (Heckel) and Plagioscion monti (Soares) in a white water lake of the Central Amazon. Amazoniana, 7(4): 465-477.

Worthmann, H.O. 1987. Vergleichende Wachstumsuntersuchungen an zwei Sciaenidenarten (Plagioscion squamosissimus Heckel und Plagioscion monti Soares) in verschiedenen Gewässertypen Zentralamazoniens. $J$. Appl. Icthtyol., 3: 124-131.

Worthmann, H.O. 1992. Aspekte der reproduktion zweier Sciaenidenarten, der pescadas Plagioscion squamosissimus (Heckel, 1864) und Plagioscion monti (Soares, 1979), Pisces, in verschiedenen Gewässertypen ZentralAmazoniens. Amazoniana, 12(1): 17-28.

Worthmann, H.O,; Oliveira, J.L. 1987. Comparative nutritional analysis of two sciaenidian species, the pescadas Plagioscion squamosissimus (Heckel) and Plagioscion monti (Soares), from different water systems of the Central Amazon. Animal Res. Developm., 25: 1-34. 
MATERIAL ADICIONAL EXAMINADO

Pachyurus junki sp. n.

(24 exemplares) - BRASIL: Amazonas: ANSP 141972, Rio Negro, confluência com Rio Branco; PARA: CAS 164327, Rio Tapajós, Santarém; CAS 32057, Rio Tapajós, Santarém; MCP 15123, Rio Tapajós, Itaituba; INPA 6762, Rio Jamanxim, Ilha Terra Preta; INPA 6860, Rio Tapajós, Pimental; INPA 15051, Rio Xingu, Altamira; INPA 15050, Rio Xingu, Senador José Porfírio; INPA 15053, Rio Xingu, Senador José Porfírio; MZUSP 36883, Cachoeira do Espelho, Rio Xingu; MZUSP 35995, Rio Fresco, Aldeia Gorotire, São Félix do Xingu; INPA 1113, Rio Tocantins, Itupiranga; MPEG 1665, Rio Tocantins, Tucuruí; INPA 10421, Rio Tocantins, Icanguí; INPA 10446, Rio Tocantins, Icanguí; INPA 10430, Rio Tocantins, Icangui; MZUSP 34074 , Rio Itacaiunas, Caldeirão, afluente do Araguaia-Tocantins; Golás: MZUSP 40665, Rio Paranã, Monte Alegre de Goiás; Mato Grosso: MZUSP 14573, Lago Dumbá Grande, Rio Araguaia; MPEG 1674, Rio Araguaia, Porto Jarbas Passarinho; MZUSP 14574, Lago Dumbá Grande, Rio Araguaia; INPA 613, Rio Aripuanã.

Pachyurus schomburgkii

Holótipo: Pachyurus schomburgkii Günther, 1860, BMNH 1849.11.8-22, 198,4 mm, comprado por Stevens, Rio Capim, Estado do Pará, Brasil.

(27 exemplares) - Venezuela:
Apure: ANSP 165491, Rio Capanaparo; Bolivar: ANSP 162800, Rio Iguapo, afluente do Orinoco; AmAZONAS: ANSP 162841, Rio Venturari; BRASIL: Roraima: MZUSP 34106, Rio Branco, praia do Xeriuini; Amazonas: ANSP 171276, Rio Negro, confluência com Rio Branco; INPA 617, Rio Negro, Anavilhanas; MZUSP 51085, Rio Solimões, Fonte Boa; MPEG 815, Rio Tefé, Jurupari; INPA 11993, Rio Uatumã; PARÁ: MZUSP 12121, Rio Trombetas, Oriximiná; MCP 15169, Rio Tapajós, Itaituba; INPA 10426, Rio Tocantins, Itupiranga; MZUSP 14556, Rio Capim, Vila Santana; RondôNIA: INPA 596, Rio Machado; Bolívia: Pando: FMNH 107112, Rio Orthon.

Plagioscion montei sp. $\mathrm{n}$.

(73 exemplares) - Brasil: RoRaIMA: MZUSP 34087, Rio Branco, Marará; AmAzonAs: INPA 9913, Rio Jaú, Igarapé Miratuca; INPA 12634, Rio Jaú, Lago Tambor Velho; INPA 9909, Rio Jaú, Igarapé Miratuca; INPA 12344, Rio Negro, Lago do Prato; INPA 12911, Rio Solimões, Ilha da Marchantaria; MZUSP 14689, Rio Madeira, Nova Olinda; INPA 12912, Rio Amazonas, Ilha do Careiro; INPA 11025, Rio Amazonas, Ilha do Careiro, Lago do Rei; INPA 2730, Rio Uatumã; USNM 316857, Rio Januari, perto de Canta Galos; PARÁ: INPA 12922, Rio Amazonas, Lago Grande de Monte Alegre; INPA 12938, Rio Amazonas, Lago Grande de Monte Alegre; INPA 10165, Rio Amazonas, Lago Grande de Monte Alegre; MZUSP 47443, Rio 
Trombetas, Cuminá; MZUSP 5693, Rio Trombetas, Boca do Lago Paru; INPA 793, Rio Trombetas, cabeceira do Lago Tapagem; MZUSP 34085, Rio Trombetas; MZUSP 5488, Rio Trombetas, Oriximiná; MZUSP 45954, Rio Tapajós, entre Itaituba e São Luis; AcrE: MZUSP 45949, Rio Tarauacá, Tarauacá; Peru: LoReto: INPA 12909, Rio Marañon, Cocha San Pablo Tipishca; UcAYalr: INPA 12891, Rio Ucayali, Cocha Caoeurahaita.

\section{Plagioscion squamosissimus}

Holótipo: Sciaena squamosissima Heckel, 1840, NMW 92124, 485,0 $\mathrm{mm}$ (material em via seca), J. Natterer leg., Forte do Rio Branco, Brasil, março de 1832.

(62 exemplares) - Venezuela: Delta Amacuro: CAS 50802, Rio Orinoco; Monagas: ANSP 149502, Cano Araguaito, Barrancas; GUÁrico: USNM 260103, Cano Falcon; APURE: ANSP 165490, Rio Capanaparo; USNM 258256, Rio Apure; ANSP 160207, Rio Meta; Bolivar: ANSP 166569, Rio Orinoco; Amazonas: ANSP 160181, Rio Orinoco; GuIANA INGLESA: CUYUNI-MAZZARUNI: AMNH 72895, Rio Mazzaruni; Essequibo: AMNH 220499, Rio Essequibo; SURINAME: NICKERIE: USNM 226087 , Rio Corantijn; ColÔMBIA: META: ANSP 140329, Rio Metica, Lago Mozambique; EQUADOR: NAPO: FMNH 101965, Rio Yasuni; FMNH 101966, Rio Aguarico; Brasil: Amazonas: INPA 5020, Rio Uaupés; INPA 3161, Rio Negro, São Gabriel da Cachoeira; INPA 12933, Rio Jaú; MZUSP 27596, Rio Solimões, Benjamin Constant;
INPA 12920, Rio Uatumã; INPA 8178, Rio Purus; MZUSP 7028, Rio Madeira; AmAPÁ: MZUSP 45948, Rio Araguari; PARÁ: INPA 12910, Rio Amazonas, Lago Grande de Monte Alegre; MZUSP 47443, Rio Trombetas, Cuminá; INPA 7136, Rio Tapajós; MZUSP 51080, Rio Amazonas; MZUSP 34132, Rio Xingu, Belo Monte; INPA 12927, Rio Tocantins, Itupiranga; ACRE: MZUSP 50535, Rio Juruá; RoNDÔNIA: MZUSP 34089, Rio Madeira, Calama; INPA 10459, Rio Mamoré; Mato Grosso: MZUSP 53720, Rio Araguaia; Mato Grosso do SUL: MNRJ 12610, Rio Tocantins; Minas Gerais: MNRJ 15328, Rio São Francisco, canal artificial, Manga; ParanÁ: MZUSP 21654, Rio Paraná, Guaíra; MZUSP 21145, Rio Paraná, abaixo de Sete Quedas; Peru: Loreto: INPA 12893, Rio Napo; BenI: AMNH 40165, Rio Mamoré.

\section{Plagioscion auratus}

Holótipo: Johnius auratus Castelnau, 1855, MNHN 7622, 198,0 $\mathrm{mm}$, Castelnau leg., Rio Ucayali, Peru.

(13 exemplares) - Venezuela: Delta Amacuro: AMNH 217675, Rio Orinoco; CAS 50803, Rio Orinoco; Guiana Inglesa: Demerara: AMNH 8235, Rio Demerara; Essequibo: AMNH 73016, Rio Cuyuni; BrasiL: Amazonas: MCZ 10865, Rio Amazonas, Óbidos; INPA 12896, Rio Amazonas, Lago Grande de Monte Alegre; MZUSP 45943, Baía de Marajó; INPA 10427, Rio Tocantins, Icangui; INPA 12934, Rio Tocantins; Peru: Loreto: CAS 147819, Rio 
Ampiyacu, próximo a Pebas.

Plagioscion surinamensis

Holótipo: Pseudosciaena surinamensis Bleeker, 1873, RMNH, 88,2 mm, Suriname.

(27 exemplares) - Colômbia: Magdalena: CAS 13890, Rio Magdalena; BrasiL: AmAZonAS: INPA 10422, Rio Solimões, Ilha da Marchantaria; AMAPÁ: MZUSP 34077, Rio Araguari, Ferreira Gomes; PARA: INPA 12924, Rio Amazonas, Lago Grande de Monte Alegre; MZUSP 34083, Rio Trombetas, Cuminá; MZUSP 3591, Lago Arari, Ilha de Marajó; MPEG 1673, Rio Goiapi, Cachoeira do Arari, Ilha de Marajó; INPA 12913, Rio Tocantins, Icangui.

\section{Plagioscion ternetzi}

Sintipos: Plagioscion ternetzi Boulenger, 1865, BMNH 32460, 2 ex., 210,6-390,0 mm, Remanso, Paraguai, C. Ternetz leg., 1895.

(18 exemplares) - BrasiL: Mato Grosso: MZUSP 51112, Rio Cuiabá, Barão de Melgaço; MATo Grosso DO SUl: MZUSP 51110, Rio Paraguai, Corumbá; PARANÁ: MZUSP 21145, Rio Paraná, abaixo de Sete Quedas; Paraguai: Neembucu: MHNG 2120.95, Lagoa do Rio Paraguai, Pilar; La Cordillera: CAS 78519, Rio Paraguai, Assunción; URuguaI: Dep. SORIANO: MZUSP 45841, Playa la Agraciada; Argentina: Missiones: MHNG 2414.27, Porto Iguaçu. 